\title{
Análisis Fractal del Electroencefalograma Durante la Vigilia en Reposo de Adultos Mayores Hidalguenses y Deterioro Cognitivo
}

\author{
Fractal Analysis of the Electroencephalogram During Rest of Older Adults from Hidalgo State and Cognitive \\ Impairment
}

Erika Elizabeth Rodríguez Torres ${ }^{\mathrm{a}, *}$, Alejandra Rosales Lagarde ${ }^{\mathrm{b}}$, Carlos Fernando Chávez Vega ${ }^{\mathrm{c}}$, José Luis Ocaña Garrido ${ }^{\mathrm{c}}$, Yair Alejandro Pardo Rosales ${ }^{c}$ y Rodrigo Silva Mota ${ }^{c}$

\author{
a Área Académica de Matemáticas y Física, Universidad Autónoma del Estado de Hidalgo, Carretera Pachuca-Tulancingo Km. 4.5, Col. Carboneras, C. P. \\ 42184, Mineral de la Reforma, Hgo., México. \\ b Cátedras CONACyT. División de Servicios Clínicos. Laboratorio de Cronobiología y Sueño, pertenecientes al Instituto Nacional de Psiquiatría Ramón de la Fuente \\ Muñiz. Calzada México-Xochimilco 101, Col. San Lorenzo Huipulco, Alcaldía de Tlalpan. C.P. 14370, Ciudad de México, México \\ ${ }^{c}$ Licenciatura en Física y Tecnología Avanzada Área Académica de Matemáticas y Física, Universidad Autónoma del Estado de Hidalgo, Carretera Pachuca- \\ Tulancingo Km. 4.5, Col. Carboneras, C. P. 42184, Mineral de la Reforma, Hgo., México.
}

\begin{abstract}
Resumen
Se sabe que el Análisis de Fluctuaciones sin Tendencia o DFA (Detrended Fluctuations Analysis, por sus siglas en inglés) de las series de tiempo biológicas como el Electrocardiograma (ECG), el Electroencefalograma (EEG) y otras, resulta ser una herramienta útil para discriminar las bioseñales de pacientes sanos o enfermos, para las cuales el coeficiente de Hurst es cercano a 1 o a 1.5, respectivamente. En particular, con el fin de conocer si la herramienta resulta útil para diferenciar las series de tiempo de Adultos Mayores (AM) con Deterioro Cognitivo (DCL) y sin Deterioro Cognitivo (sDCL), se registró el EEG y el Electromiograma (EMG) a dos sujetos, uno con DCL o sDCL, durante la condición de vigilia con ojos cerrados y se calcularon los DFA por medio de una interfaz programada en Python. Durante la condición de reposo con ojos cerrados, el sujeto con DCL presentó exponentes de Hurst más altos en regiones frontales en comparación con el sujeto sDCL. Se concluye que el DFA aporta información cuantificable sobre la localización y mecanismos subyacentes al DCL que pueden servir para monitorear el curso del DCL en el AM.
\end{abstract}

\section{Palabras Clave:}

EEG, Series de Tiempo, Fractalidad, Exponente de Hurst, Deterioro Cognitivo Leve, Adulto Mayor.

\begin{abstract}
It is known that the Detrended Fluctuations Analysis or DFA of biological time series like the Electrocardiogram (ECG), the Electroencephalogram (EEG) and others, turns out to be a useful tool to discriminate the biosignals of healthy or sick patients, for which the Hurst coefficient is close to 1 or 1.5, respectively. In order to corroborate if this tool was useful to differentiate time series of Older Adults (OAs) with Mild Cognitive Impairment (MCI) and without Mild Cognitive Impairment or Control (CTRL), EEGs and Electromiograms (EMGs) of two OAs, one with MCI and one CTRL were registered during the eyes-closed condition. DFAs were calculated by means of a Phyton application interface. It was observed that the subject with MCI had higher values in frontal regions than the CTRL subject. It is concluded that the DFA provides quantifiable information on the location and mechanisms underlying MCI that can serve to help to monitor the course of MCI in OAs.
\end{abstract}

Keywords:EEG,

Time series, Fractal, Hurst Exponent, Mild Cognitive Impairment, Elderly.

\section{Introducción}

El Deterioro Cognitivo Leve (DCL) es un síndrome neuropsiquiátrico cuyo aumento anual en México resulta alarmante (Gutiérrez-Robledo y cols., 2017). El Adulto Mayor (AM) con DCL tiene problemas de memoria, de lenguaje, en su pensamiento o juicio y esos problemas son más significativos que los cambios del envejecimiento normal (Petersen, 2004). No obstante, este síndrome no es tan grave como la demencia, padecimiento que interfiere significativamente con la vida diaria. La enfermedad de Alzheimer es la demencia más frecuente en la actualidad y se calcula que en el año 2050 llegará a ser la causa de

*Autor para la correspondencia: erikart@uaeh.edu.mx

Correo electrónico: erikart@uaeh.edu.mx (Erika Elizabeth Rodríguez Torres), alexiaro@ rocketmail.com (Alejandra Rosales Lagarde), fchavez1503@gmail.com (Carlos Fernando Chávez Vega), jluisocana2519@gmail.com (José Luis Ocaña Garrido), AlEX_YaPp@ hotmail.com (Yair Alejandro Pardo Paredes), Mota.rm97@gmail.com (Rodrigo Silva Mota) 
la mayoría de las demencias hasta en 3 millones de personas en nuestro país (Gutiérrez-Robledo y cols., 2017).

El DCL puede aumentar el riesgo de padecer demencia en el futuro, aunque también es interesante que algunas personas con DCL mejoren con el tiempo, aunque su porcentaje sea muy pequeño.

En un estudio previo (Rosales-Lagarde y cols., 2018), se analizó la transición del sueño llamado No de Movimientos Oculares Rápidos (NMOR) al sueño propiamente con MOR y se observó que los exponentes de Hurst, exponentes que se obtienen gracias a los Análisis de Fluctuaciones sin Tendencia (DFA, por sus siglas en inglés), fueron mayores en sujetos con DCL en comparación con los sujetos sin Deterioro Cognitivo Leve (sDCL). Estas diferencias se observaron en regiones frontales y temporales, áreas que están implicadas en procesos cognitivos que estarían siendo reactivadas especialmente durante la etapa de sueño MOR. Es decir, que las actividades que llevamos a cabo durante nuestro tiempo de estar despiertos se ven "reflejadas" o "integradas" en nuestro cerebro durante el sueño, por lo que el estudio del sueño representa un marcador del estado funcional de nuestras neuronas (Rosales-Lagarde y cols., 2018).

Por medio de métodos lineales es posible predecir la transición de la normalidad al DCL y del DCL hacia la demencia durante la condición de ojos cerrados (Prichep, 2007). Sin embargo, para hacer esta distinción no se han empleado métodos no lineales que podrían resultar ser ventajosos dado que las señales utilizadas son no lineales y caóticas. Por lo tanto, aunque en el presente escrito no se presenta la comparación estadística con aquellos valores obtenidos durante el sueño NMOR y MOR de varios sujetos (Rosales-Lagarde y cols., 2018), en este trabajo se calcularon los exponentes de Hurst de los adultos mayores durante la condición de vigilia con ojos cerrados de dos sujetos, uno con DCL y otro sDCL. En un futuro, es posible pensar en realizar registros longitudinales con métodos no lineales para monitorear a adultos mayores con DCL cuya evolución pueda o no transitar hacia la demencia. Además, gracias a este tipo de investigaciones básicas, es factible avanzar en la perspectiva de que se vuelvan útiles para los especialistas de salud en su proceso de toma de decisiones. En un futuro, un diagnóstico más completo del AM permitiría a los especialistas de salud implementar intervenciones farmacológicas y/o no farmacológicas en aquellos pacientes con DCL.

\section{Materiales y Métodos}

\subsection{Método Experimental}

La administración de la batería de pruebas para medir memoria, atención y otros procesos psicológicos se realizó tanto en el Centro Gerontológico Integral (CGI) de la Colonia Punta Azul en la ciudad de Pachuca de Soto, Hidalgo como en el Laboratorio de Sueño, Emoción y Cognición bajo la dirección de la Dra. Alejandra Rosales Lagarde perteneciente a Cátedras CONACyT y comisionada al Área Académica de Gerontología del Instituto de Ciencias de la Salud de la Universidad Autónoma del Estado de Hidalgo. Cada AM que cumplió con los criterios sin depresión, sin ansiedad, con o sDCL-, se registró con posterioridad en las condiciones abajo descritas.

Se colocaron electrodos en el cuero cabelludo de los dos sujetos. Se utilizó el MEDICID-5 con 26 amplificadores, 19 electrodos de plata clorurada, los cuales fueron colocados de acuerdo al Sistema Internacional 10-20. Se presentan los resultados de los análisis de DFA de las siguientes señales: FP1 (localizado en la parte fronto-polar izquierda), FP2 (fronto-polar derecha), F3 (frontal izquierda), F4 (frontal derecha), F7 y F8 (laterales frontales izquierdo y derecho, respectivamente), C3 y C4 (parte central izquierda y derecha, respectivamente), EMG (músculos del mentón). Los lóbulos de las orejas cortocircuitados fueron usados como referencias con excepción del registro del EMG, ya que éstos se referenciaron de forma bipolar.

Las señales de EEG y EMG se registraron bajo dos condiciones, una con ojos abiertos cuya duración fue de 5 minutos y la siguiente donde a cada uno de los AM se les pidió cerrar y abrir los ojos durante 20 segundos varias veces seguidas (ver Figura 1). En la Figura 1 se observa el registro de C3. Para este artículo sólo se analizó la primera parte correspondiente al reposo con ojos cerrados. Esos 5 minutos fueron divididos en períodos de 30 segundos y para cada período la señal fue segmentada y sometida al DFA.

\subsection{Método Matemático.}

El análisis de los datos se basó en los conceptos sobre el color del ruido y el análisis de fluctuaciones sin tendencia (Rodríguez y cols., 2011), que se expondrán a continuación.

\subsubsection{El color del ruido}

El color del ruido, se deriva de una analogía entre el espectro de onda del sonido con el de la luz. Es decir, si el patrón de ondas de sonido del ruido azul se tradujera en ondas de luz, la luz resultante sería azul, y así con los otros colores.

La práctica de nombrar a los tipos de ruido después de los colores comenzó con el ruido blanco. Cuando se obtiene una línea recta en coordenadas logarítmicas de un espectro de potencias se tiene una interpretación novedosa. Dado que la abscisa en el espectro de potencias es la frecuencia, una línea recta horizontal querría decir, en analogía con lo que sucede con el espectro visible, que todas las frecuencias están presentes y con la misma amplitud. El ruido blanco se puede escuchar en la estática de los radios antiguos o al tocar notas distintas sin coordinación ni patrón.

Continuando con la analogía, si la recta en el espectro de potencias tiene una pendiente unitaria y negativa, entonces, todas las frecuencias están presentes, pero dominan las frecuencias bajas, las que en el espectro visible corresponden al rojo. En este caso se dice que la señal es ruido color de rosa. Éste es un caso muy estudiado pues no sólo coincide con el experimento de Zipf, sino que es el escenario que permite la criticalidad autoorganizada.

El permitir la criticalidad autoorganizada, quiere decir que los sistemas dinámicos formados por un gran número de componentes no lineales, tienen una tendencia espontánea a organizarse a sí mismos es estados críticos de equilibrio dinámico donde ocurren fluctuaciones de todos los tamaños. El ruido blanco a menudo es usado en la investigación acústica porque tiene un poder constante por octava (intervalo de ocho grados entre dos notas de la escala musical), por lo que está bien adaptado a la respuesta audible humana.

También de gran relevancia es el caso de una pendiente de -2 pues se ha demostrado que coincide con una serie de tiempo correspondiente a un movimiento browniano. Su nombre viene de Robert Brown y no tiene relación alguna con el color, sin embargo, es conocido y llamado como ruido café.

El caso extremo de que en el espectro de potencias existe una recta de pendiente de $-\infty$ también tiene sentido pues se puede interpretar como una delta de Dirac en una frecuencia particular. Esto es, un movimiento periódico.

A continuación, se define un método fractal que asocia el color del ruido con un exponente de autosimilitud o exponente de Hurst. 


\subsection{Análisis de fluctuaciones sin Tendencia}

El DFA es un método desarrollado en la universidad de Harvard por un equipo conformado por físicos, matemáticos y médicos (Peng y cols., 1994). Este método puede ser empleado para la detección de auto-similitudes o fractalidad, intrínsecas a las señales biológicas y además evita la detección espuria de pseudoauto-similitudes cuyo origen puede ser artefactual (por tendencias extrínsecas). La metodología del DFA se describe a continuación. Se inicia con una serie de tiempo $x(i)$ la cual es discreta $i=1,2, \ldots, N$. Después se calculan las sumas parciales de la serie de datos corregidas por la media:

$$
y(k)=\sum_{i=1}^{k}(x(i)-\bar{x}),
$$

donde $\bar{x}=\frac{1}{N} \sum_{i=1}^{N} x(i)$ es el valor promedio de la señal.

Se mapea la serie de tiempo a un proceso autosimilar. El siguiente paso es medir la escala vertical de características de la serie de tiempo integrada, lo cual se obtiene dividiendo la integral de la serie de tiempo en ventanas de igual tamaño $(n)$. Para cada una de las ventanas de datos se ajusta un modelo lineal por mínimos cuadrados, el cual representa la tendencia local en cada ventana. El valor de la ordenada $y$ de la línea recta se denota como:

$$
y_{n}(k) * \text {. }
$$

Para eliminar la tendencia de la serie de tiempo integrada $y(k)$, para cada ventana se sustrae la tendencia local lineal $y_{n}(k)$. Para cada tamaño de ventana $n$ la escala característica para las fluctuaciones en la serie integrada y sin tendencia es:

$$
\mathrm{F}(n)=\sqrt{\frac{1}{N} \sum_{k=1}^{N}\left(y(k)-y_{n}(k)\right)^{2}} .
$$

Al graficar los valores de $\log n$ versus los valores de $\log \mathrm{F}(n)$, se observa una relación lineal la cual indica la presencia de una potencia logarítmica, esto es:

$$
\mathrm{F}(n): n^{\alpha} .
$$

Así que, el exponente escalar $\alpha$, o exponente de Hurst representa las fluctuaciones ${ }^{\dagger}$ y se puede aproximar como una pendiente de la línea relacionada $\log \mathrm{F}(n)$ y $\log n$.

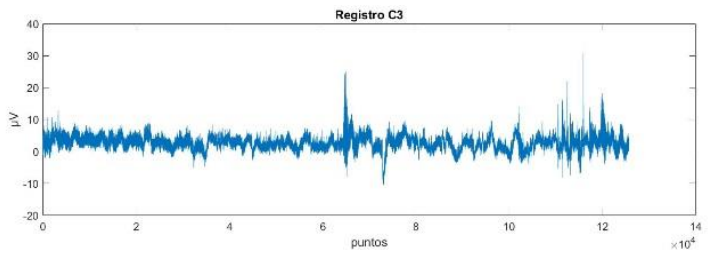

Figura 1. Registro de Electroencefalograma (EEG) de la región central izquierda (C3). La primera parte corresponde a la señal de un adulto mayor en reposo con los ojos cerrados. A la mitad del registro se le dio la instrucción de abrir y cerrar los ojos varias veces. El análisis de fluctuaciones sin tendencia (DFA, por sus siglas en inglés) se calculó para varios perídos correspondientes a 30 segundos y se obtuvieron exponentes de Hurst o $\alpha$. El caso de $\alpha=1$ representa al ruido rosa en registros de personas normales. La ocurrencia de un evento al azar es un $\alpha=0.5$, color blanco o señal aleatoria y un valor $\alpha=1.5$, se relaciona con color de ruido café en registros de personas con problemas de salud.

* más general, $y_{n}(k)$ puede representar la coordenada - $y$ - de grado $m$ del polinomio de ajuste. Esto es muy importante si se desea eliminar no sólo las tendencias constantes o términos lineares, sino también tendencias de
El DFA revela la correlación de largo alcance en aparentemente series de tiempo no estacionarias. Un valor de $\alpha$ mayor que 0.5 indica la presencia de una correlación de largo alcance.

El caso de $\alpha=1.0$ ha llamado la atención a físicos y biólogos porque corresponde al ruido rosa o registros de personas normales. La ocurrencia de un evento al azar es un $\alpha=0.5$, color blanco o señal aleatoria y un valor $\alpha=1.5$, se relaciona con color de ruido café en registros de personas con problemas neurológicos o cardiacos (Rosales-Lagarde y cols., 2018, Kobayashi \& Musha, 1982 y Peng y cols., 1993).

Se utilizó una interfaz gráfica, donde se programó en Python el DFA (Rodriguez-Torres y cols., 2019). Esta interfaz cuenta con una herramienta que permite segmentar los registros (Figura 1) de EEG en períodos de 30 segundos. Esto se realizó para las señales FZ, FP1, FP2, F3, F4, F7, F8, C3, C4, y EMG.

\section{Resultados}

Para el cálculo del DFA, en la interfaz se cargan los segmentos y se introduce como parámetro el grado del polinomio de ajuste (igual a 1). La interfaz muestra la gráfica $\log \mathrm{F}(n)$ vs $\log n$, el exponente de Hurst y el coeficiente de calidad del ajuste, $\mathrm{R}^{2}$ (Figura 2).

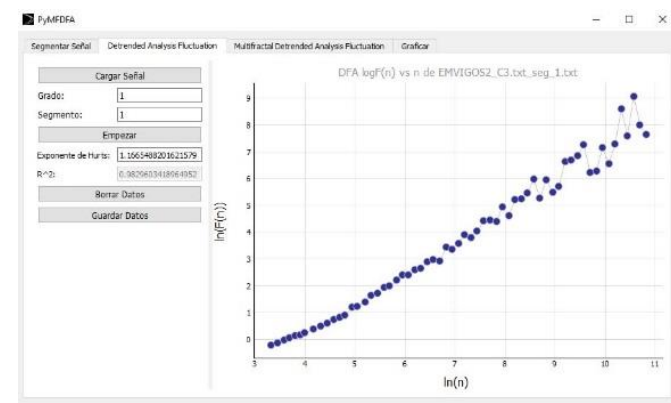

Figura 2. Se ilustra la interfaz donde se realizó el Análisis de Fluctuaciones sin Tendencia (DFA, por sus siglas en inglés) del primer segmento de 30 segundos del registro electroencefalográfico en la región central izquierda $(C 3)$ mostrada en la Figura 1. Se puede observar que se obtiene un exponente de Hurst o $\alpha$ $=1.16$ con un ajuste del $98 \%\left(\mathrm{R}^{2}\right)$.

Los segmentos por registro para el sujeto sDCL fueron 8 .

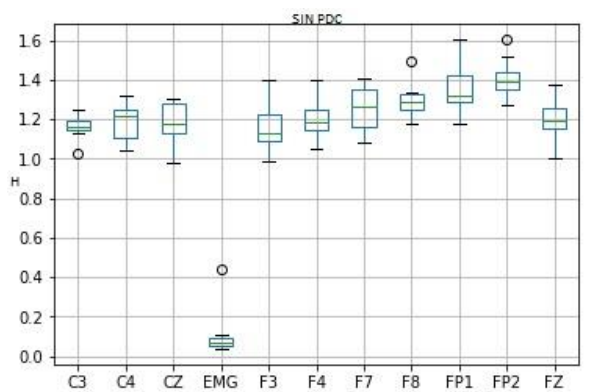

Figura 3. Los boxplots muestran los Análisis de Fluctuaciones sin Tendencia (DFA, por sus siglas en inglés) de un participante sin deterioro cognitivo $(\mathrm{sDCL})$. Promedio \pm desviación estándar de $\mathrm{DFA}_{\mathrm{C} 3}=1.160+0.065$, $\mathrm{DFA}_{\mathrm{CZ}}=1.177+0.114, \mathrm{DFA}_{\mathrm{EMG}}=0.112+0.135, \mathrm{DFA}_{\mathrm{F} 3}=1.158+0.129$, $\mathrm{DFA}_{\mathrm{F} 4}=1.200 \pm 0.104, \mathrm{DFA}_{\mathrm{F} 7}=1.256 \pm 0.115, \mathrm{DFA}_{\mathrm{F} 8}=1.297 \pm 0.096$ $\mathrm{DFA}_{\mathrm{FP} 1}=1.362+0.140, \mathrm{DFA}_{\mathrm{FP} 2}=1.404+0.109, \mathrm{DFA}_{\mathrm{FZ}}=1.197+0.107$. Las

orden mayor. En este caso se refiere como sigue DFAm, con $m=0,1,2, \ldots$, representa el grado del polinomio asociado. 
letras y números contiguos corresponden a los canales analizados. Las abreviaturas se refieren a la región frontal central (FZ), frontopolar izquierda (FP1), frontopolar derecha (FP2), frontal izquierda (F3), frontal derecha (F4), frontal lateral izquierda (F7), frontal lateral derecha (F8), central izquierda (C3), central derecha (C4), y Electromiograma (EMG).

Los valores del DFA para el AM sDCL fueron menores (Figura 3) comparados con aquellos del participante con DCL (Figura 4). El valor de DFA $\mathrm{F}_{3}$ del AM sDCL fue de $1.158+0.129$ en contraste con un participante con DCL de $1.331 \pm 0.058$.

Algo similar se observó en el registro simétrico en F3 donde para el participante sDCL se obtuvo un $\mathrm{DFA}_{\mathrm{F} 4}=1.200 \pm 0.104$, y con $\mathrm{DCL}, \mathrm{DFA}_{\mathrm{F} 4}=1.355 \pm 0.062$.

El participante $\mathrm{sDCL}$ tuvo un $\mathrm{DFA}_{\mathrm{F} 7}=1.256 \pm 0.115$ que se aproxima más al ruido rosa versus el participante con un $\mathrm{DFA}_{\mathrm{F} 7}=$ $1.458 \pm 0.064$ más cercano al ruido café.

También en el registro contralateral a F7 se encontraron valores mayores para el participante con DCL en comparación con aquel sDCL: DFA $_{\mathrm{F} 8}=1.396 \pm 0.093, \quad \mathrm{DFA}_{\mathrm{F} 8}=1.297 \pm 0.096$ respectivamente.

Además, el valor de DFA del EMG tanto para el AM sDCL como para el AM con DCL tuvo valores muy pequeños. El valor máximo registrado para el AM sDCL fue de $\mathrm{DFA}_{\mathrm{EMG}}=0.439 \mathrm{y}$ para el AM con DCL tuvo un valor de $\mathrm{DFA}_{\mathrm{EMG}}=0.435$. Ambos valores tienen un valor cercano a 0.5 que corresponde al ruido blanco que indica aleatoriedad.

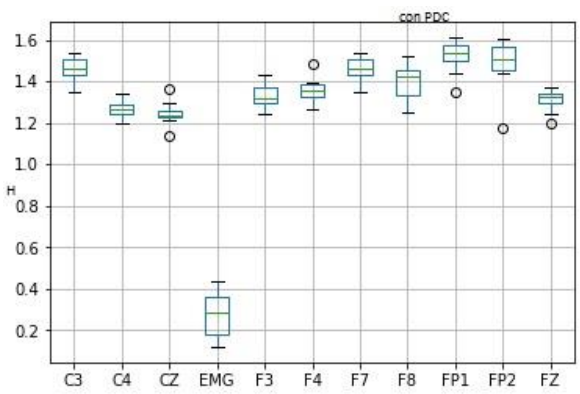

Figura 4. Los boxplots muestran Análisis de Fluctuaciones sin Tendencia (DFA, por sus siglas en inglés) de un participante con deterioro cognitivo (DCL). Promedio + desviación estándar de $\mathrm{DFA}_{\mathrm{C} 3}=1.458+0.064, \mathrm{DFA}_{\mathrm{C} 4}=$ $1.267+0.040, \mathrm{DFA}_{\mathrm{CZ}}=1.243+0.058, \mathrm{DFA}_{\mathrm{EMG}}=0.271+0.115, \mathrm{DFA}_{\mathrm{F} 3}=$ $1.331 \pm 0.058, \mathrm{DFA}_{\mathrm{F} 4}=1.355 \pm 0.062, \mathrm{DFA}_{\mathrm{F} 7}=1.458 \pm 0.064, \mathrm{DFA}_{\mathrm{F} 8}=1.396$ $+0.093, \mathrm{DFA}_{\mathrm{FP} l}=1.521+0.080, \mathrm{DFA}_{\mathrm{FP} 2}=1.485+0.124, \mathrm{DFA}_{\mathrm{FZ}}=1.309+$ 0.054 . Las letras y números contiguos corresponden a los canales analizados. Las abreviaturas se refieren a la región frontal central (FZ), frontopolar izquierda (FP1), frontopolar derecha (FP2), frontal izquierda (F3), frontal derecha (F4), frontal lateral izquierda (F7), frontal lateral derecha (F8), central izquierda (C3), central derecha (C4), y Electromiograma (EMG).

\section{Discusión}

En este trabajo se encontraron valores mayores del exponente de Hurst en regiones frontales en el sujeto con DCL en comparación con el sujeto sDCL. Los resultados preliminares presentados en este trabajo son consistentes con otros previos (Rosales-Lagarde y cols., 2018), ya que las personas con DCL presentaron una tendencia al ruido café, en especial en la parte frontal, región especializada en el procesamiento de la toma de decisiones, el cual tuvo un exponente de Hurst aproximadamente igual a $\alpha=1.5$. Otros estudios han relacionado el color de ruido café con algunos problemas cardiacos (Kobayashi y Musha, 1982; Peng y cols., 1993). En cambio, aquellas personas saludables presentan más cercanía al ruido rosa que tiene un exponente de Hurst aproximadamente igual a $\alpha=1.0$. Esto es muy interesante porque por primera vez se presentan estos hallazgos durante la vigilia con menos de 5 minutos analizados en reposo con ojos cerrados puesto que en los estudios previos estos análisis se llevaron a cabo en registros cuya duración fue muy larga y durante la transición de una etapa de sueño a otra. En cuanto al color del ruido de la señal obtenida en el músculo, éste coincidió con los estudios mencionados de Rosales-Lagarde y cols. (2018), ya que el ruido de esta señal se observó de color blanco. Este color presenta un exponente de Hurst aproximadamente igual a $\alpha=0.5$, lo que indica que la señal registrada fue aleatoria. Estos resultados parecen prometedores por las coincidencias antes descritas, sin embargo, deben tomarse con precaución porque en un futuro próximo se completarán los análisis de más sujetos para tener la posibilidad de compararlos y poder concluir sobre los procesos fisiológicos involucrados.

\section{Referencias}

Gutiérrez-Robledo, L. M. G., García-Peña, M.C., Roa-Rojas, P. A. \& Martínez-Ruiz, A. (eds.) (2017). La Enfermedad de Alzheimer y otras demencias como problema nacional de salud. Documento de postura. CONACyT, Academia Nacional de Medicina de México, México.

Kobayashi, M., \& Musha, T. (1982). 1/f fluctuation of heartbeat period. IEEE transactions on Biomedical Engineering, (6), 456-457.

Peng, C. K., Mietus, J., Hausdorff, J. M., Havlin, S., Stanley, H. E., \& Goldberger, A. L. (1993). Long-range anticorrelations and non-Gaussian behavior of the heartbeat. Physical review letters, 70(9), 1343.

Peng, C. K., Buldyrev, S. V., Havlin, S., Simons, M., Stanley, H. E., \& Goldberger, A. L. (1994). Mosaic organization of DNA nucleotides. Physical review e, 49(2), 1685.

Petersen, R. C. (2004). Mild cognitive impairment as a diagnostic entity. Journal of internal medicine, 256(3), 183-194.

Prichep, L.S. (2007). Quantitative EEG and electromagnetic brain imaging in aging and in the evolution of dementia. Ann N Y Acad Sci., 1097, 156-167. Rodríguez E.E., Hernández-Lemus, E., Itzá-Ortiz, B.A., Jiménez, I., Rudomín, P. (2011). Multichannel Detrended Fluctuation Analysis Reveals Synchronized Patterns of Spontaneous Spinal Activity in Anesthetized Cats. PLoS ONE 6(10): e26449. doi:10.1371/journal.pone.0026449

Rodriguez-Torres, E.E., Contreras-Alcalá, F., Miramontes, P., Itzá-Ortiz, B., Quezada-Tellez, L.A., Martínez-Alcalá, C.I., Enciso-Alva, J.C., GarcíaMuñoz, V., Vázquez-Tagle, G., Rosales-Lagarde, A. (2019). An interated function system implemented on a Phyton application as a tool to detect dementia and Mild Cognitive Impairment during Rapid Eye Movement Sleep and Rest. Sleep, 42 (Supplement_1): A381-A381. Doi: 10.1093/sleep/zsz067.946

Rosales-Lagarde, A., Rodriguez-Torres, E. E., Itzá-Ortiz, B. A., Miramontes, P., Vázquez-Tagle, G., Enciso-Alva, J. C., \& Lopez-Noguerola, J. S. (2018). The Color of Noise and Weak Stationarity at the NREM to REM sleep transition in Mild Cognitive Impaired subjects. Frontiers in psychology, 9 . 